\title{
PRÉSENCE DE LA MÉDECINE VÉTÉRINAIRE DANS LA REVUE MAROC-MÉDICAL (1921-1956)
}

\author{
PRESENCE OF VETERINARY MEDICINE IN MAROC-MEDICAL JOURNAL
} (1921-1956)

\author{
Jamal HOSSAINI-HILALI ${ }^{1} \mathcal{G}^{\text {Fatima }}$ YAACOUB ${ }^{2}$
}

(Mémoire accepté le 25 avril 2021)

\begin{abstract}
RÉSUMÉ
Parmi les publications scientifiques éditées au Maroc durant la période du Protectorat, la revue Maroc-Médical occupait une place de choix. Cette étude se propose de rappeler l'itinéraire de cette revue et d'évaluer, en termes quantitatif et qualitatif, les articles d'intérêt vétérinaire qui y ont été publiés durant la période 1921-1956. Ainsi, 379 numéros de la revue ont été publiés et seules les conditions économiques de la Deuxième Guerre mondiale ont ralenti le rythme de parution. Durant la période d'étude, 298 articles d'intérêt vétérinaire ont été publiés soit une moyenne de 8,2 articles par année. Ces articles, axés principalement sur la pathologie infectieuse, ont été écrits par 97 auteurs dont 66 étaient des vétérinaires. Parmi ceux-ci, les principaux sont Velu, Zottner, Placidi, Bailly, Belle, Jeaume, Miègeville, et Grimpret. Dès son lancement, cette publication mensuelle s'est caractérisée par sa dualité comme une revue à la fois scientifique et professionnelle, ouverte aux contributions des médecins et des vétérinaires. Une sorte de plateforme "One Health » avant l'heure.
\end{abstract}

Mots-clés : Revue Maroc-Médical, médecine vétérinaire, recherche scientifique, Protectorat, Maroc.

\begin{abstract}
-ABSTRACT
Among the scientific publications edited in Morocco during the period of the Protectorate, the journal Maroc-Médical occupied a prominent place. This study proposed to recall the historical itinerary of the journal and to evaluate, in quantitative and qualitative terms, the articles of veterinary interest that were published during the period 1921-1956. Thus, 379 issues of the journal were published and only the economic conditions of the Second World War slowed the rate of publication. During the period of the study, 298 articles of veterinary interest were published with an average of 8.2 articles per year. Those articles, focused mainly on infectious pathology, were written by 97 authors, among them, 66 were veterinarians and the main ones were Velu, Zottner, Placidi, Bailly, Belle, Jeaume, Miègeville, and Grimpret. Since its launch, this monthly publication has been characterized by its duality as a as scientific and professional journal open to physicians and veterinarians contributions: a kind of "One Health" platform ahead of time.

Key-Words: "Maroc-Médical" journal, veterinary medicine, scientific research, Protectorate, Morocco.
\end{abstract}

\section{INTRODUCTION}

La recherche vétérinaire moderne au Maroc utilisant une approche expérimentale a débuté avec l'instauration du Protectorat franco-espagnol (1912-1956). Cette recherche était nécessaire pour diverses raisons. L'exploitation des ressources animales pour la production de la viande, laine, peau et œufs nécessitait la connaissance de l'environnement pathologique marocain afin d'établir des stratégies efficaces pour la prévention et le traitement des maladies animales. De même, l'utilisation des équidés dans le transport des troupes et des marchan- dises demandait une maitrise de la pathologie locale. Enfin, l'éradication des zoonoses nécessitait la compréhension des mécanismes de contagion et de transmission des maladies entre l'animal et l'homme.

Les résultats de cette recherche ont été publiés dans les revues scientifiques et les bulletins des sociétés savantes au Maroc et en France (La Société de Médecine et d'Hygiène du Maroc, La Société des Sciences naturelles du Maroc, La Société vétérinaire de Zootechnie du Maroc, La Société de Pathologie exotique, La Société centrale de Médecine vétérinaire devenue L'Académie vétérinaire de France, L’Académie des Sciences).

(1) Professeur de Physiologie animale, Institut agronomique et vétérinaire Hassan-II, BP 6202, Rabat, Maroc ; j.hossaini@iav.ac.ma

(2) Vétérinaire praticienne libérale, Sidi Addi, Province d'Ifrane, Maroc ; yaacoubfatimavet@gmail.com 
Parmi les publications éditées au Maroc, la revue Maroc-Médical occupait une place de choix. Depuis sa création dans sa forme mensuelle régulière en 1921, elle s'est caractérisée par sa dualité. Revue à la fois scientifique et professionnelle, elle est ouverte aux médecins et aux vétérinaires, considérant la médecine de l'homme et des animaux, comme des sciences médicales proches et imbriquées.

Cette étude se propose de rappeler l'historique de la revue Maroc-Médical dans le domaine de la presse médicale scientifique au Maroc et d'évaluer, en termes quantitatif et qualitatif, les articles d'intérêt vétérinaire qui y ont été publiés. Nous nous limiterons à la période du Protectorat car les publications de cette période ne sont pas répertoriées dans les bases de données bibliographiques et restent donc inconnues pour une grande majorité des utilisateurs.

\section{APPROCHE MÉTHODOLOGIQUE}

Un balayage des numéros de la revue depuis sa parution mensuelle régulière en $1921\left(\mathrm{~N}^{\circ} 1\right)$ jusqu'à la fin du Protectorat en 1956 ( $\left.{ }^{\circ} 379\right)$ a été réalisé dans divers sites (Bibliothèque nationale du Royaume du Maroc à Rabat, Bibliothèque du laboratoire vétérinaire de Casablanca, Bibliothèque nationale de France). Malgré ces efforts, nous n'avons pas pu avoir accès à 23 numéros datant de 1939 à 1946 ; ce qui représente 6\% de la totalité des numéros de la période d'étude. Les numéros non consultés sont rares car ils ont été réalisés sur du papier de qualité médiocre, se détériorant facilement, vu les conditions de la guerre.

Les publications répertoriées sont celles ayant un intérêt vétérinaire. Elles s'intéressent à la pathologie animale, la zootechnie et l'hygiène des denrées alimentaires d'origine animale. Parallèlement, d'autres publications traitent de la pathologie commune à l'homme et à l'animal ainsi que de la pathologie vectorielle avec l'animal comme vecteur ou réservoir (cas du paludisme). Parfois, ces publications étaient incluses dans une partie vétérinaire distincte appelée selon les années "partie vétérinaire", "chronique vétérinaire", "pathologie comparée" ou "articles originaux". Souvent, ces articles étaient incorporés à l'ensemble sans être répertoriés dans une rubrique distincte.

Dans cette étude, seules les publications considérées comme une contribution originale ont été comptabilisées. C'est-à-dire un article scientifique signé par un ou plusieurs auteurs et rapportant une expérience scientifique, un cas clinique ou une mise au point bibliographique.

Durant la période de l'étude (1921-1956), la revue Maroc-Médical a publié des numéros spéciaux consacrés à la médecine vétérinaire et à l'élevage. Les articles de ces numéros spéciaux n'ont pas été inclus dans l'étude quantitative pour ne pas biaiser les résultats mais feront l'objet d'une présentation à part.

\section{APERÇU HISTORIQUE}

\section{Naissance}

La revue Maroc-Médical est née le 1 janvier 1920 dans une forme embryonnaire qui était plutôt une sorte de bulletin de liaison édité par l'Union médicale de Casablanca qui avait pour principale mission la défense des intérêts professionnels des médecins. Après une période d'essai de ce bulletin et profonde réflexion, la décision a été prise par son initiateur, le Dr Emile Speder de le transformer en une revue scientifique et professionnelle. Ainsi, le premier numéro de la revue dans sa forme régulière mensuelle va être lancé le 15 novembre 1921 (Lepinay, 1967).

Speder a tracé dans l'éditorial du premier numéro l'objectif de la revue et sa feuille de route. Dans ce long éditorial, il énonça le programme de Maroc-Médical en précisant «qu'elle ne sera pas l'organe d'une union professionnelle, d'une société scientifique ou d'un groupe restreint de médecins. Son but est de devenir l'organe scientifique et professionnel de tous les médecins du Maroc : civils et militaires, libres, fonctionnaires ou à contrat, français ou étrangers, des villes et $d u$ bled. Son ambition est d'être utile à tous et partout...sa seule règle sera de ne publier jamais que des articles ne contenant aucune attaque personnelle contre des personnalités ou des collectivités» (Speder, 1921).

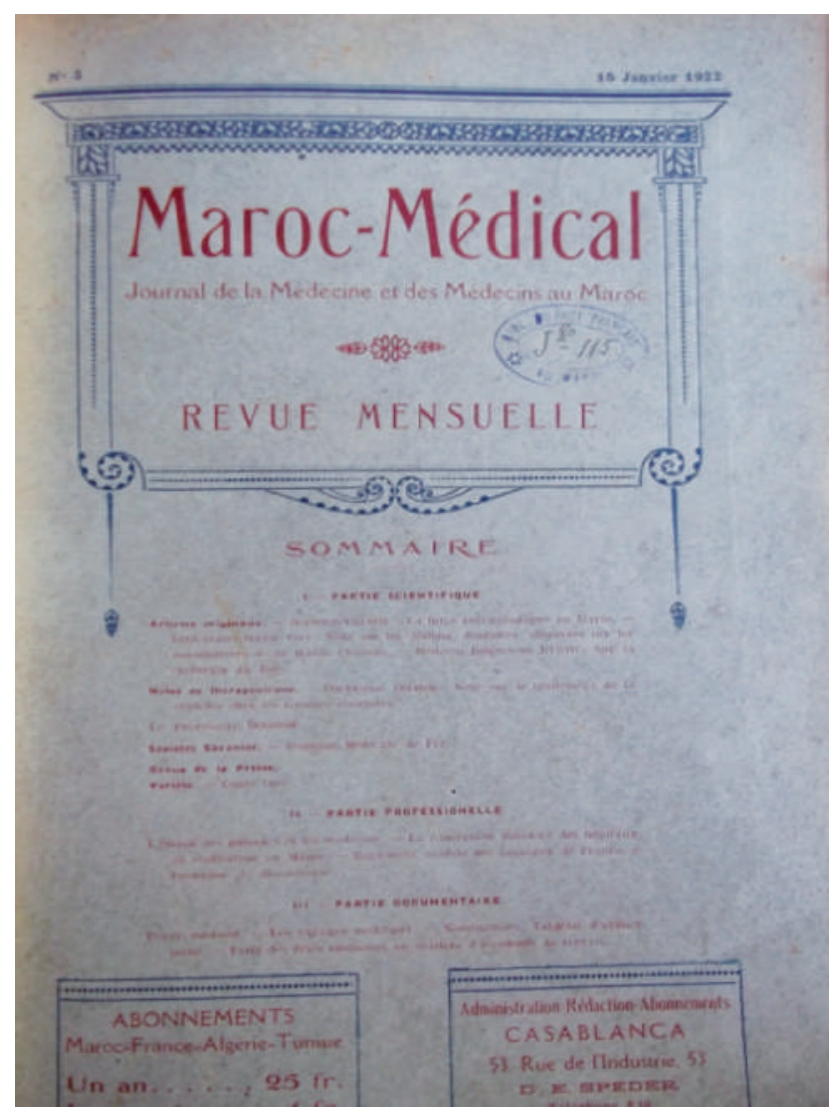

Figure 1 : Première de couverture du numéro 3 (15 janvier, 1922) de la revue Maroc-Médical.

\section{Appellation}

Le titre principal de la revue Maroc-Médical n'a pas changé depuis son lancement. Il était accompagné d'un sous-titre qui variait avec les années et qui traduisait une volonté de recherche d'une identité thématique et d'une cible de lecteurs. Ainsi, le premier sous-titre qui figurait dans la page de couverture des premiers numéros était "Journal de la Médecine et des Médecins au Maroc " (Figure 1) ou " Journal des Médecins du Maroc ». En 1936, ce sous-titre deviendra « Journal de Médecine et Pathologie Comparée du Maroc ». A partir du numéro de janvier 1957, juste après l'indépendance du Maroc, ce sous-titre 
va être éliminé complètement pour être remplacé par la traduc tion en langue arabe de Maroc-Médical «at-Tib fi al-Maghrib » et à partir du numéro de novembre 1958, on y ajouta le sous-titre en langue espagnole «Marruecos Médico».

\section{Rythme de parution}

Une fois lancée, la revue Maroc-Médical va être publiée à une cadence mensuelle le 15 de chaque mois. Cet objectif va être atteint et maintenu avec succès durant plusieurs années (22 sur 35). La Deuxième Guerre mondiale et la crise économique qui lui succéda (rareté et cherté du papier) n'ont pas interrompu la parution de la revue mais ont ralenti de moitié son rythme. Si on ne compte pas les deux numéros parus à la fin de 1921, nous pouvons affirmer que 377 numéros de la revue Maroc-Médical ont été publiés de 1922 à 1956, soit une moyenne de 10,8 numéros par année.

\section{Directeurs}

Entre 1921 et 1956, trois directeurs se sont succédé à la tête de la revue Maroc-Médical. Il s'agit des Drs Emile Speder, Eugène Lepinay et Jean Chenebault.

\section{Emile Speder}

C'est le fondateur de la revue Maroc-Médical. Lauréat de la Faculté de Médecine de Bordeaux, il débarqua à Casablanca en 1916 où il fut chargé de l'organisation du service central d'électroradiologie et de physiothérapie de l'armée. Une fois la Première Guerre mondiale terminée, il quitta l'armée pour s'installer à son compte à Casablanca (Lepinay, 1967). Esprit dynamique et organisateur avisé, il avait fait construire un petit immeuble à Casablanca dans lequel il a réuni son cabinet de radiologie, les bureaux de la revue Maroc-Médical et sa résidence personnelle (Dubois-Roquebert, 1967).

Speder était la force motrice de Maroc-Médical et il en avait conscience. Il s'occupa de la revue en tant que directeur et rédacteur-en-chef depuis son lancement en 1921 jusqu'à 1947, date à laquelle, il s'est senti fatigué et a demandé à son collaborateur Eugène Lepinay de prendre la relève. Il décéda un an après en décembre 1948 (Lepinay, 1967).

\section{Eugène Lepinay}

C'est en 1947 qu'Eugène Lepinay remplaça Emile Speder à la direction de la revue. Succession amplement méritée car Lepinay fut pour Speder un conseiller éclairé et dynamique. C'est un dermato-vénérologue qui a acquis, après sa sortie de la Faculté de Médecine de Paris, une solide expérience professionnelle dans les hôpitaux de Paris. Il fut mobilisé au Maroc où sa spécialité était très demandée car les maladies vénériennes et les dermatoses constituaient un véritable fléau (Lepinay, 1967).

Lepinay est le fils d'un vétérinaire, Lidoire Lepinay qui contribua un demi-siècle auparavant à la création de la Société de pathologie comparée et à sa revue éponyme (Hubscher, 1999). Suivant les traces de son père, Lepinay créa en 1923, la Société de médecine et d'hygiène du Maroc qui réunissait des médecins, des vétérinaires, des pharmaciens et des chimistes. La revue Maroc-Médical sera la tribune privilégiée pour la publica- tion des comptes rendus de cette société savante (Lepinay, 1967). Cette liaison familiale, entre Lepinay père et fils, expliquerait pourquoi la revue Maroc-Médical a pris la même orientation que la Revue de Pathologie Comparée éditée en France. Les deux revues ne se limitaient pas aux préoccupations et travaux scientifiques des médecins humains dans une vision corporatiste stérile mais ont pris le gage, dès leur départ, de devenir le point de rencontre des différentes corporations médicales (médecins, vétérinaires, pharmaciens) dans le seul objectif est l'amélioration de la santé de l'homme et de l'animal. Une mise en pratique du concept "One Health " un siècle avant sa vulgarisation. Dans plusieurs articles, les pathologies humaine et animale sont traitées d'une manière globale en analysant la relation homme-animal (Placidi, 1953) ou entre animaux domestiques et la faune sauvage (Remlinger \& Bailly, 1933). Parfois on n'hésite pas à tester chez l'animal des traitements ou prophylaxies efficaces chez l'homme comme le cas de la vaccination par le BCG (Balozet, 1930). Aussi, quelques syndromes communs aux animaux et à l'homme sont présentés ensemble pour faire ressortir leurs similitudes et différences (Martin, 1952).

\section{Jean Chenebault}

C'est en 1948 que Jean Chenebault, pneumologue de formation, a pris la direction de la revue et la transforma en une société ayant une personnalité civile. Grand esprit, dynamique et enthousiaste, il donna une large envergure à la revue avec l'aide de Lucienne Faivre qui assurait le secrétariat et la comptabilité. La diffusion de la revue augmenta et le Maroc-Médical eut sa "Maison " comprenant bureau, bibliothèque et salle de lecture à la nouvelle adresse sise au $\mathrm{N}^{\circ} 287$, rue de la Liberté. Jean Chenebault continua l'administration de Maroc-Médical après l'indépendance du Maroc (Lepinay, 1967).

\section{Adresse légale}

Au début de sa création, l'adresse légale de la revue était sise au $N^{\circ} 53$, rue de l'Industrie à Casablanca. En 1930, cette adresse changea au 16, rue d'Alger qui correspondait à l'adresse du cabinet de radiologie du Dr Speder. En 1948, le siège légal de la revue se déplaça, pour quelques années, au $\mathrm{N}^{\circ} 1$, rue Baudin, à Casablanca. En 1951, l'adresse légale est fixée au N²87, rue de la Liberté à Casablanca qui correspondait à l'adresse de la « Maison » Maroc-Médical.

\section{ANALYSE DES ARTICLES D'INTERÊT VÉTÉRINAIRE}

\section{Évolution annuelle}

Il est à rappeler que le premier article d'intérêt vétérinaire publié dans la revue Maroc-Médical, a été signé par Henri Velu (numéro 3, janvier 1922), qui occupait alors le poste de directeur du Laboratoire de Recherches du Service de l'Elevage de Casablanca. Cet article était intitulé "Note sur des lésions dentaires observées sur les mammifères de la haute Chaouïa" inaugurant ainsi une série d'articles sur la fluorose dans les zones phosphatières du Maroc. 
Sur 356 numéros consultés, presque la moitié soit 182, ne contenait aucun article d'intérêt vétérinaire comme défini dans notre méthodologie. Pour le reste des numéros (soit 174), 298 articles d'intérêt vétérinaires ont été recensés. Leur évolution annuelle est représentée par la Figure 2. Nous constatons que le nombre d'articles par année montre une nette tendance à l'augmentation jusqu' à 1937, puis diminue avec le déclenchement de la Deuxième Guerre mondiale qui fut une période difficile pour la revue. Par la suite, le nombre d'articles va montrer une tendance à l'augmentation vers les années 1950.

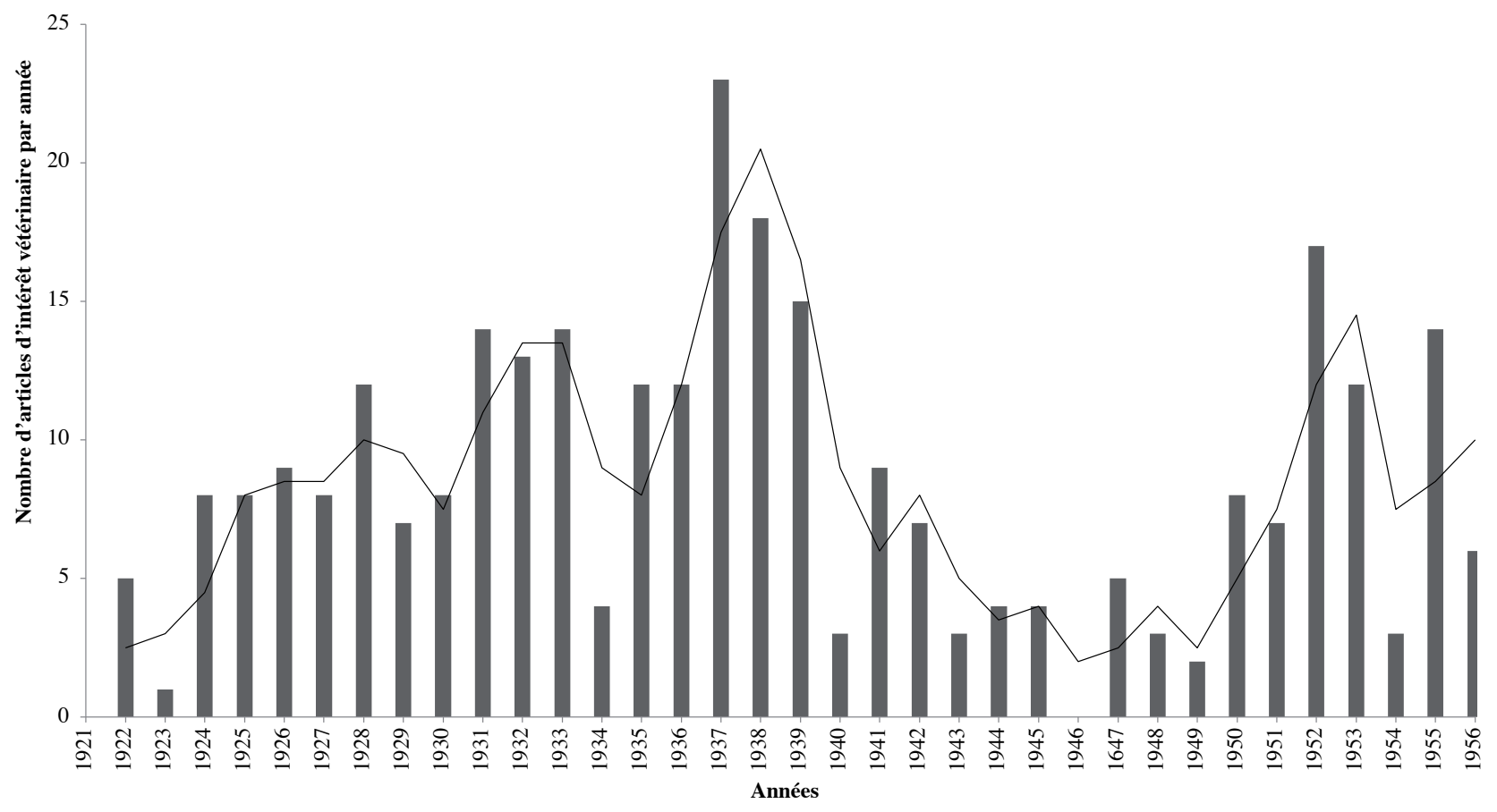

Figure 2 : Évolution annuelle des articles d'intérêt vétérinaire publiés dans la revue Maroc-Médical entre 1921-1956.

Sur toute la période de l'étude (1921-1956), la moyenne était de 8,2 articles par année avec un maximum observé durant l'année 1937 avec 23 articles. Une autre façon d'évaluer l'importance des articles d'intérêt vétérinaire collectés est de les relativiser par rapport à l'ensemble des articles publiés. Dans les années 1920 et 1930, les articles d'intérêt vétérinaire ont représenté 14\% des articles totaux avec un maximum de $21 \%$ observé en 1931. Par la suite, ce pourcentage tend à diminuer pour se situer à $10 \%$ dans les années 1950.

\section{Répartition par auteur}

Les auteurs qui ont contribué à la publication des articles collectés (298 en total) étaient au nombre de 97 dont 66 (soit $68 \%$ ) étaient des vétérinaires. Dans le tableau 1, les dix premiers auteurs sont classés par ordre d'importance en assumant que le premier est l'auteur principal en cas de plusieurs signataires. On constate facilement que ces dix auteurs ont publié un total de 206 articles, soit environ 70\% de la totalité du corpus de l'étude. Cependant, ce classement des auteurs est à nuancer car leur durée de séjour au Maroc ne fut pas la même. Deux auteurs se démarquent du groupe, il s'agit de Paul Remlinger (1871-1964) et Henri Velu (1887-1973) :

\section{Paul Remlinger (1871-1964)}

Médecin et biologiste français dont l'œuvre scientifique est immense. Il a travaillé au début des années 1900 à l'Institut antirabique de Constantinople dont il fut directeur. En 1913, il a intégré l'Institut Pasteur de Tanger nouvellement créé, où il est devenu un spécialiste du virus de la rage et de la vaccination antirabique. Il a été secondé, selon une tradition bien connue de l'Institut Pasteur, par 3 vétérinaires qui sont Joseph Séjournant de 1913-1914 qui a été tué à la guerre, Bel de 1922-1924 et Jaques Bailly de 1925 à 1955 (Dedet, 2000). Remlinger s'est penché sur l'étude de la rage expérimentale, de sa clinique chez différentes espèces animales et de la vaccination antirabique telle que pratiquée à cette époque par l'usage de la moelle de lapin séchée. L'ensemble de ces travaux a été récompensé par plusieurs prix notamment le prix Dagnan-Bouveret de l'Académie des Sciences. En 1957, il a été mis à la retraite, contre sa volonté, à l'âge de 84 ans. Il quitta alors son logement de fonction mais continua de vivre à Tanger jusqu'à son décès en 1964.

\section{Henri Velu (1887-1973)}

Diplômé de l'École vétérinaire d'Alfort en 1909, Henri Velu a opté pour la carrière militaire. Il est affecté au Maroc, comme directeur du Laboratoire de Recherches du Service de l'Élevage de Casablanca qu'il dirigera pendant 25 ans avant de quitter le Maroc en 1938. Vétérinaire de talent, fin microbiologiste, travailleur, il n'est pas un sujet d'intérêt vétérinaire au Maroc où Velu n'ait apporté sa signature : maladies infectieuses, parasitaires, carences alimentaires, intoxications et zootechnie. Il est considéré comme le pionnier de la médecine vétérinaire moderne au Maroc (Milhaud et al. Coll, 2005). 
Tableau 1 : Classement des principaux auteurs des articles d'intérêt vétérinaire publiés dans la revue Maroc-Médical (1921-1956).

\begin{tabular}{lcll} 
Auteur & $\begin{array}{l}\text { Nombre } \\
\text { d'articles }\end{array}$ & Affiliation & Titre \\
1. Paul Remlinger & 70 & Institut Pasteur, Tanger & Médecin \\
\hline 2. Henri Velu & 48 & $\begin{array}{l}\text { Laboratoire de Recherches, } \\
\text { Casablanca }\end{array}$ & Vétérinaire \\
\hline 3. Gustave Zottner & 18 & $\begin{array}{l}\text { Laboratoire de Recherches, } \\
\text { Casablanca }\end{array}$ & Vétérinaire \\
\hline 4. Louis Placidi & 15 & $\begin{array}{l}\text { Institut de Biologie animale, } \\
\text { Rabat }\end{array}$ & Vétérinaire \\
\hline 5. Louis Martin & 12 & Institut Pasteur, Casablanca & Médecin \\
\hline 6. Jaques Bailly & 10 & Institut Pasteur, Tanger & Vétérinaire \\
\hline 7. Gustave Belle & 10 & $\begin{array}{l}\text { Laboratoire de Recherches, } \\
\text { Casablanca }\end{array}$ & Vétérinaire \\
\hline 8. Maurice Jeaume & 10 & Circonscription d'élevage & Vétérinaire \\
\hline 9. Joseph Miègeville & 7 & Circonscription d'élevage & Vétérinaire \\
\hline 10. Jean Grimpret & 6 & Circonscription d'élevage & Vétérinaire
\end{tabular}

\section{Répartition par axe de recherche}

Chaque article collecté a été classé dans une seule rubrique même si parfois, il s'est trouvé à cheval entre plusieurs catégories. Dans ce cas, nous avons opté pour l'axe le plus dominant. Les résultats sont présentés dans le Tableau 2. Nous remarquons que presque les $3 / 4$ des articles publiés concernaient la pathologie médicale vétérinaire avec une prépondérance de la pathologie infectieuse. Il est à noter que les articles consacrés à la virologie constituaient presque la moitié de cette catégorie (116 articles sur 218 , soit $53 \%$ ) avec une prédominance de la rage (65 articles). En effet, les épidémies d'origine virale causaient des pertes importantes dans le cheptel. En 1946, les autorités du Protectorat ont pris la décision de créer à Rabat l'Institut aphteux du Maroc, consacré initialement à l'étude de la fièvre aphteuse mais qui va englober par la suite l'étude des principales pathologies virales. Cet institut a été dirigé pendant de longues années par le Dr Louis Placidi (Hossaini-Hilali, 2015). Il a été renommé Institut de Biologie animale (IPA) et ses locaux vont être partagés entre le Ministère de l'Agriculture (service de l'élevage) et celui de la Santé publique (service de la transfusion sanguine).

Concernant la pathologie bactérienne, celle-ci était dominée par la tuberculose et la brucellose vu le risque de transmission à l'homme (Zottner, 1939 ; Aldebert, 1956). Quant aux maladies parasitaires, elles étaient axées sur les hémopathogènes (theilériose, anaplasmose, babésiose) avec des essais de vaccination non concluants et des traitements réussis à base de la gonacrine (Grimpret, 1943).

En matière d'HIDAOA (Hygiène et Industrie des Denrées Alimentaires d'Origine Animale), la thématique principale était l'étude de la qualité hygiénique du lait cru, l'efficacité des traitements thermiques et les risques de transmission à l'homme de la tuberculose et la brucellose par le lait (Belle, 1937).
Tableau 2 : Répartition par thématique des articles d'intérêt vétérinaire publiés dans la revue Maroc-Médical (1921-1956).

\begin{tabular}{ccc} 
Thématique & Nombre d'articles & Pourcentage du total \\
Pathologie médicale & 218 & $73 \%$ \\
\hline Virologie & 116 & \\
Bactériologie & 28 \\
Parasitologie & 30 \\
Mycologie & 5 \\
Cancérologie & 11 \\
Autres & 28
\end{tabular}

\begin{tabular}{lll}
\hline Pharmacologie-Physiologie & 21 & $7 \%$ \\
\hline Toxicologie & 18 & $6 \%$ \\
\hline HIDAOA & 15 & $5 \%$ \\
\hline Reproduction-Obstétrique & 10 & $3 \%$ \\
\hline Zootechnie-Alimentation & 5 & $2 \%$ \\
\hline Autres & 11 & $4 \%$ \\
\hline Total & 298 & $100 \%$ \\
\hline
\end{tabular}

\section{NUMÉROS SPÉCIAUX CONSACRÉS A LA MÉDECINE VÉTÉRINAIRE}

La revue Maroc-Médical a publié plusieurs numéros spéciaux dont 4 étaient consacrés à la médecine vétérinaire et à l'élevage. Ces numéros spéciaux étaient en réalité des comptes rendus de congrès, conférences ou journées vétérinaires organisés au Maroc. Nous les citerons par ordre chronologique.

\section{Deuxièmes journées médicales et vétérinaires marocaines}

Organisées en avril 1928 pendant les vacances de Pâques, ces journées ont fait l'objet de comptes rendus publiés dans 3 numéros : un numéro hors-série en janvier 1929 et deux numéros normaux en avril et juin 1929. Les Deuxièmes Journées médicales et vétérinaires marocaines furent un succès marquant, illustré par la notoriété des conférenciers dont nous citerons :

- Charles Nicolle, médecin et microbiologiste français, lauréat du prix Nobel de physiologie ou médecine en 1928, qui a donné une conférence sur la présence au Maroc du spirochète de la fièvre récurrente espagnole ; maladie endémique au Maroc comme dans toute l'Afrique du Nord et transmise à l'homme par le pou de corps.

- Albert Calmette qui a présenté une étude sur le vaccin BCG développé avec son confrère, le vétérinaire Camille Guérin.

- Jules Bordet de la Faculté de Médecine de Bruxelles qui a traité de la variabilité microbienne.

- Henri Velu et Louis Balozet, les deux vétérinaires du Laboratoire de Recherches du Service de l'Élevage à Casablanca qui ont présenté les résultats histopathologiques et physiologiques de la greffe testiculaire réalisée chez le cheval, l'âne et le mouton. Ces expériences ont été réalisées, pour confirmer ou infirmer le 
rajeunissement prétendument obtenu, par le Dr Serge Voronoff de Paris, grâce à cette technique. La conclusion des deux vétérinaires était sans équivoque : la majorité des animaux transplantés ne retrouvèrent par leur vigueur sexuelle de jeunesse. Au microscope, le tissu greffé se transforme en " un tissu cicatriciel avec des cellules inflammatoires» et la greffe testiculaire était une "glande d'illusion".

\section{Numéro spécial consacré à la médecine vétérinaire}

C'est le N²92 de septembre 1949. La conception et la mise en œuvre de ce numéro a été coordonnée par Louis Martin de l'Institut Pasteur de Casablanca. Une première partie a été consacrée à la situation de l'élevage au Maroc et aux circonstances de création du Service de l'élevage, sous l'impulsion des vétérinaires militaires débarqués avec le corps expéditionnaire, sous la direction du vétérinaire-colonel Monod. Dans la deuxième partie, nous trouvons des études concernant les affections à virus au Maroc à savoir la pseudo-rage, la maladie de Carré, et un essai de vaccination contre la peste porcine. Dans une troisième partie, des données relatives à l'antibiothérapie et la prophylaxie de la tuberculose ont été présentées par les vétérinaires Velu, Hintermann et Goret. Ils ont attiré l'attention sur la coopération qui s'imposait entre médecin et vétérinaire dans le vaste domaine des zoonoses et de la pathologie comparée.

\section{Journées vétérinaires marocaines}

Elles ont été organisées en avril 1952 et leurs comptes rendus ont été publiés par la revue Maroc-Médical dans son numéro de juillet 1952. Nous n'avons pas pu consulter ce numéro pour en restituer une synthèse.

\section{Premières journées de l'élevage au Maroc}

Elles ont été organisées par la jeune Société vétérinaire de Zootechnie du Maroc en avril 1954 qui était présidée à l'époque par le Dr vétérinaire Gustave Belle du Laboratoire de Casablanca. Ces journées se sont déroulées sous une forme itinérante dans les villes de Fès, Meknès, Rabat et Casablanca et se sont étalées sur une semaine du 5 au 11 avril 1954 (Belle, 1954). C'était une occasion pour les autorités du Protectorat et du Service de l'élevage en particulier de mettre le point sur les acquis et les résultats obtenus en matière d'élevage, et de tracer les lignes directrices pour orienter le développement futur du secteur. Aussi, ces journées ont connu, en plus de la participation des vétérinaires, agronomes, zootechniciens et éleveurs du Maroc, celle des hautes autorités scientifiques dans le domaine de l'élevage et la zootechnie. Nous citerons, parmi les conférenciers, le Pr Letard, titulaire de la chaire de zootechnie à l'école vétérinaire d'Alfort ; le Pr Ladrat, titulaire de la chaire de zootechnie à l'École d'Agriculture de Grignon ; le Pr Leroy, titulaire de la chaire de zootechnie à l'Institut agronomique de Montpellier, Le Pr Cordiez, titulaire de la chaire de zootechnie de l'Ecole vétérinaire de Bruxelles et le Dr Quaedwlig, directeur honoraire des Services vétérinaires des Pays-Bas. Les conférences données par ces invités de marque se sont focalisées sur des aspects d'actualité en élevage bovin comme l'alimentation, l'acclimatation des animaux au milieu et les caractéristiques de la race Pie noire hollandaise.

Il faut rappeler que ces journées se sont passées alors que le Sultan du Maroc, Feu Mohammed V était en exil à Madagascar et que les villes et la campagne marocaines connaissaient des troubles et une résistance accrue pour le retour du Sultan et l'indépendance du pays.

Dans son allocution de clôture, le Dr Belle a souligné la relation intrinsèque liant la médecine vétérinaire et la zootechnie qui doivent cheminer ensemble pour une meilleure amélioration de la productivité. Il écrivait que « Médecine vétérinaire et zootechnie reposent sur les mêmes connaissances et sont étroitement liées. Bien plus, la formation du pathologiste est indispensable au zootechnicien. Comment en effet entreprendre l'amélioration d'un troupeau à partir d'animaux déficients ou malades ? Ce serait bâtir sur un sol mouvant "(Belle, 1954). Cette synergie entre les sciences vétérinaires et la zootechnie a été agréablement illustrée par l'appellation de l'association que Belle présidait : La Société vétérinaire de Zootechnie du Maroc.

\section{CONCLUSION}

Dans ce travail, nous avons rappelé l'itinéraire historique de la revue Maroc-Médical dans le domaine de la presse médicale scientifique au Maroc et avons recensé et évalué les articles d'intérêt vétérinaire qui y ont été publiés durant la période 1921-1956.

Durant cette période, 379 numéros, hormis les numéros spéciaux, ont été publiés, signifiant que la cadence de publication mensuelle a été atteinte durant plusieurs années. Les numéros consultés contenaient 298 articles d'intérêt vétérinaire qui englobaient tous les domaines des sciences vétérinaires. La majorité (soit 73\%) des articles vétérinaires publiés concernaient la pathologie médicale vétérinaire dont la moitié (soit 53\%) était consacrée à la pathologie infectieuse virale et en premier lieu à la rage. Les deux grands contributeurs de la médecine vétérinaire dans la revue Maroc-Médical sont le médecin Paul Remlinger, directeur de l'Institut Pasteur de Tanger et le vétérinaire Henri Velu, directeur du Laboratoire de Recherches du Service de l'Élevage de Casablanca.

On peut conclure que la matière scientifique d'intérêt vétérinaire publiée dans la revue Maroc-Médical est impressionnante par sa quantité et par la diversité des thématiques abordées. Elle doit être préservée et vulgarisée auprès des vétérinaires (étudiants, chercheurs, praticiens) pour une meilleure connaissance de l'histoire des sciences vétérinaires au Maroc. A ce titre, il serait intéressant de publier cette matière scientifique sous forme numérique pour faciliter l'accès à l'information. 


\section{BIBLIOGRAPHIE}

- Aldebert J-C. Considérations sur la prophylaxie de la tuberculose animale. Maroc-Médical. 1956 ; 368 : 19-27.

- Balozet A. Essai au Maroc de vaccination des bovidés contre la tuberculose par le BCG. Maroc-Médical. 1930 ; 99 : 312-313.

- Belle G. État actuel de l'hygiène du lait au Maroc. Maroc-Médical. 1937 ; 180 : 216-219.

- Dedet J-P. Les instituts Pasteur d'outre-mer : cent vingt ans de microbiologie française dans le monde. Éditions de L'Harmattan, Paris, France ; 2000.

- Dubois-Roquebert H. Éditorial. Maroc-Médical. 1967 ; 500 : 135-141.

- Grimpret J. Traitement de la theilériose bovine. Maroc-Médical. 1943 ; 231 : 61-63.

- Hossaini-Hilali J. Des vétérinaires au Maroc sous le Protectorat français. Adrar Editions, Rabat, Maroc ; 2015.

- Hubscher R. Les maîtres des bêtes : les vétérinaires dans la société française (XVIIIe-XXe siècle). Éditions Odile Jacob, Paris, France ; 1999.

- Lepinay E. Souvenirs. Maroc-Médical. 1967 ; $500: 143-147$.

- Martin L.A. De quelque virus hépato-neurotropes du chien et de l'homme, leur point de contact. Maroc-Médical. 1952 ; 326 : 630-638.

- Milhaud C. \& Coll J-L. Henri Velu (1887-1973), pionnier de la médecine vétérinaire au Maroc : une introduc- tion. Bulletin de la Société Française d'Histoire de la Médecine et des Sciences Vétérinaires. 2005 ; 4 : 104-120.

- Placidi L. Vue d'ensemble sur l'influence et les manifestations de quelques facteurs climatiques dans la biologie et la pathologie générales et comparées de l'homme et de l'animal. Maroc-Médical. 1953 ; 342 : 1212-1230.

- Remlinger P. \& Bailly J. La transmission de la maladie d'Aujeszky au chacal et à l'épervier. Maroc-Médical. 1933 ; ○ 137 : 578-579.

- Zottner G. Les brucelloses animales. Maroc-Médical. 1939 ; 206 : 288-292.

- Speder E. Éditorial. Maroc-Médical. $1921 ; 1: 3-7$. 